\title{
Disrupted Expression of Circadian Clock Genes in Patients with Bronchial Asthma
}

This article was published in the following Dove Press journal:

Journal of Asthma and Allergy

Hung-Chen Chen (D)
Yung-Che Chen (D)
Tsu-Nai Wang
Wen-Feng Fang
Ya-Chun Chang
Yu-Mu Chen'
I-Ya Chen ${ }^{2}$
Meng-Chih Lin ID
Ming-Yu Yang iD ${ }^{2,4}$
'Division of Pulmonary and Critical Care
Medicine, Department of Medicine, Chang
Gung Memorial Hospital-Kaohsiung
Medical Center, Chang Gung University
College of Medicine, Kaohsiung, Taiwan;
${ }^{2}$ Graduate Institute of Clinical Medical
Sciences, College of Medicine, Chang Gung
University, Tao-Yuan, Taiwan; ${ }^{3}$ Department
of Public Health, College of Health Science,
Kaohsiung Medical University, Kaohsiung,
Taiwan; ${ }^{4}$ Department of Otolaryngology,
Kaohsiung Chang Gung Memorial Hospital,
Kaohsiung, Taiwan

Kaohsiung, Taiwan
Correspondence: Ming-Yu Yang

Graduate Institute of Clinical Medical

Sciences, College of Medicine, Chang

Gung University, No. 259, Wenhua Ist

Road, Guishan Dist., Taoyuan City, 33302,

Taiwan

Email yangmy@mail.cgu.edu.tw

Meng-Chih Lin

Division of Pulmonary and Critical Care

Medicine, Department of Medicine,

Kaohsiung Chang Gung Memorial

Hospital, No. 123, Dapi Road Niaosong

Dist., Kaohsiung City, 8330I, Taiwan

Email mengchih@cgmh.org.tw
Purpose: Circadian clock is synchronized to the 24-hour day by the daily light-dark cycle and proper function of circadian rhythm is essential for many physiological processes. Disruption of circadian rhythm can affect disease processes and influence disease severity, treatment responses, and even survivorship. In this retrospective case-controlled study, we tried to explore whether expression of circadian clock genes was disturbed in patients with bronchial asthma.

Patients and Methods: We performed real-time quantitative reverse transcriptasepolymerase chain reactions to examine the expression of the nine core circadian clock genes (BMAL1, CK1E, CLOCK, CRY1,CRY2, PER1, PER2, PER3, and TIM) in total leukocytes of peripheral blood collected at chest clinics from 120 patients with asthma and 60 health individuals.

Results: Expression levels of the nine circadian clock genes were significantly different between patients and healthy individuals, but not associated with the asthma control status. We also noted the difference of PER3 expression in asthmatic patients with and without nocturnal symptoms. In well-controlled asthmatics, expression of BMAL1, CK1E, CLOCK, $C R Y 1, C R Y 2$, and PER1 was significantly lower in patients with nocturnal symptoms than those without nocturnal symptoms. However, in not well-controlled asthmatics, expression of only BMAL1, CK1E, PER1, and PER2 was significantly different between patients with and without nocturnal symptoms. Binary logistic regression analysis selected BMAL1, CKIE, $P E R 3$, and TIM as independent factors for bronchial asthma and ROC curves showed the combined expression of these four genes enhanced the capability of predicting asthma (AUC $=0.924 ; 95 \% \mathrm{CI}=0.875-0.958 ; P<0.001$ ).

Conclusion: Our results showed altered expression of circadian clock genes in patients with bronchial asthma and down-regulated PER3 in patients with nocturnal symptoms. Altered expression of circadian clock genes was also observed in asthmatics with or without nocturnal symptoms in well- or not well-controlled subgroups. Combined expression of $B M A L 1, C K I E, P E R 3$, and TIM could be a potential predictor for bronchial asthma.

Keywords: bronchial asthma, nocturnal symptom, circadian clock genes, PER3

\section{Introduction}

Bronchial asthma is characterized by variable airflow limitation and chronic airway inflammation with a history of respiratory symptoms such as wheeze, shortness of breath, chest tightness, and cough that vary over time and in intensity. ${ }^{1}$ It is an important characteristic of asthma that the appearance of symptoms displays a circadian rhythm ${ }^{2}$ and frequently exacerbates in the early morning hour at around 4:00AM, which is also an occurrence time of sudden death. ${ }^{3}$ It has been reported that about $74 \%$ of asthmatic patients are awakened by asthma symptoms at least 
once per week and around $40 \%$ of patients experienced symptoms nightly. ${ }^{3}$ Nocturnal asthma is typically defined as the difference in forced expiratory volume in 1s (FEV1) and peak expiratory flow rate between daytime and night is greater than $15 \%$ in patients with asthma. ${ }^{4}$ This overnight decline of lung function causes a more severe form of the disease and is associated with airway inflammation and hyper-responsiveness. ${ }^{5}$

Circadian rhythms are intrinsic biological oscillations with an interval near 24 hours operated in mammals by the circadian clock system. ${ }^{6}$ Molecular circadian systems are found at the cellular level by an autoregulatory transcriptional-translational feedback loop operated with at least nine circadian clock genes including PER1, PER2, PER3, CLOCK, CRY1, CRY2, BMAL1, CK1E, and TIM. ${ }^{7}$ The circadian presentations of asthma, like airflow limitation and airway hyper-responsiveness, were associated with chronic inflammation and it may be secondary to changes of the parasympathetic nervous system and endocrine system at night. ${ }^{8,9}$ Circadian clock genes also regulate inflammatory responses and contribute to lung inflammation, fibrosis, immunity, and glucocorticoid response. ${ }^{10-12}$ Therefore, increasing evidence demonstrates the molecular circadian clock is important in the pathogenesis of asthma.

The clock in the monocyte of peripheral blood mononuclear cells (PBMC) controlled the rhythmic expression of downstream genes ${ }^{13}$ and influenced on innate immunity including gating of pattern-recognition receptors response, clock-controlled cytokine response and recruitment to tissues. $^{13-15}$ Rhythmic cytokine secretion from monocytes has been reported in humans under sleep/ wake and laboratory conditions. ${ }^{16,17}$ In addition, a rhythm of the adaptive immune response was measured as antibody production at different times of the day. ${ }^{18}$ That hints that either $\mathrm{T}$ or $\mathrm{B}$ lymphocyte response and the adaptive immune system was associating with the molecular circadian clock. ${ }^{19,20}$ Some asthmatic patients with nocturnal symptoms had a significantly higher number of alveolar eosinophils at 4:00AM than at 4:00PM, and the increase of alveolar eosinophils correlated with a decrease in FEV1. ${ }^{3,21}$ Also, the number of macrophages, neutrophils and CD4 T-cells in the bronchoalveolar lavage fluid from patients with asthma were higher at 4:00AM than at 4:00PM, and the increase of CD4 T-cells also correlated with decreased FEV1. ${ }^{3,21}$

Recently, several studies found circadian clock underpins allergic reaction and possibly influences the periodicity of allergic disease. ${ }^{22-24}$ The induction of asthma was associated with markedly increased inflammation in the lungs in mice lacking Bmall expression in myeloid cells, and a higher numbers of eosinophils and increased IL-5 levels in the lung and serum. ${ }^{25}$ Since altered circadian rhythm deregulates epithelial barrier function and immune function, the two fundamental biological aspects of allergic disease, the circadian clock is strongly related to allergic disease.

For the substantial associations of circadian presentations between asthma and allergic diseases, and for the lack of reports about expression of circadian clock genes in patients with asthma, we therefore hypothesized that the expression of circadian gene expression might be disrupted in asthmatic patients and correlated with nocturnal symptoms of asthmatic patients. To test our hypothesis, we constructed a retrospective casecontrolled study focusing on expression of circadian clock genes of PBMCs in patients with bronchial asthma and healthy individuals and to explore the correlation of circadian clock gene expression between difference control status and clinical presentation in these patients. In addition, we also tried to build a prediction model for asthma by finding the expression of a specific circadian clock gene in PBMCs.

\section{Patients and Methods}

\section{Patients, Healthy Subjects, and Samples}

We conducted this retrospective case-controlled study between January 2019 and December 2019 at Kaohsiung Chang Gung Memorial Hospital (K-CGMH), Taiwan. The design of this study adhered to the principles of the Declaration of Helsinki. This study was approved by the Institutional Review Board (IRB) of the CGMH Ethical Committee (IRB No. 201801642B0A3C501). Written informed consent was obtained from all participants.

Adult asthmatic patients were recruited from the Division of Pulmonary and Critical Care Medicine of K-CGMH and the Division of Chest Medicine of Kaohsiung Medical University Hospital (KMUH) in Taiwan from January 2013 to January 2015. The candidates had symptoms such as episodic breathlessness, wheezing, cough, and chest tightness and the diagnosis of asthma according to the Global Initiative for Asthma (GINA) guidelines, with or without reversible airway from spirometry that showed an increase in FEV1 of at least 
$12 \%$ and at least $200 \mathrm{~mL}$ from the prebronchodilator value. Those who met the previous criteria were regarded to have asthma if they had had at least one asthma exacerbation or needed use of asthma prescription in the 12 months before the clinics visit. Patients were excluded if they were physician-diagnosed with chronic airway obstruction, emphysema, tuberculosis, or cancer.

We obtained residual cDNA samples of PBMCs and collected clinical parameters and outcomes from 120 patients with asthma (60 men and 60 women, aged 25-73) and 60 age- and gender-matched healthy individuals (30 men and 30 women, aged 20-73). All the enrolled subjects were aged 18 years or older. Pregnant women, psychiatric patients, and individuals taking sleep pills were excluded. Subjects enrolled in this study did not experience shift work or jet lag 1 week before PB collection. The study participants were screened for eligibility and recruited from the pulmonary clinics and health examination center of K-CGMH and KMUH during the period of January 2013 through January 2015. Clinical characteristics including gender, age, smoking habits, body weight, body mass index (BMI), body fat, body height, waist, IgE, FEV1, PEF (peak expiratory flow rate), and asthma control test (ACT) were recorded. We defined the well-controlled and poor-controlled asthma as ACT scores equal to or more than 20 and below 20, respectively. Patients with nocturnal symptoms were identified in the third question of ACT, with a score lower than 5, which means asthma symptoms (wheezing, coughing, shortness of breath, chest tightness, or pain) have woken patients up at night or earlier than usual in the morning during the past 4 weeks.

\section{Analysis of Expression of Circadian Clock Genes}

The expression of the nine circadian clock genes and $A C T B$ gene (as endogenous reference control) was analyzed using real-time quantitative reverse transcriptase-polymerase chain reaction (qRT-PCR) as previously described. ${ }^{26,27}$ The relative threshold cycle $(\Delta \mathrm{C} t)$ of the circadian clock gene was obtained by normalizing the threshold cycle of the circadian clock gene to the $A C T B$ gene of the same sample, ie, $\Delta \mathrm{C} t=$ ( $\mathrm{C} t$ of circadian clock gene $-\mathrm{C} t$ of $A C T B$ gene). Thus, a higher $\Delta \mathrm{C} t$ value represents a lower expression level and a higher $-\Delta \mathrm{C} t$ value represents a higher expression level.

\section{Statistical Analysis}

We used MedCalc software (MedCalc Software Ltd, Ostend, Belgium) and Graph Pad Prism 7.04 (GraphPad, San Diego, CA, USA) for all statistical analyses. The sample size was estimated by $G$ power software (Supplementary Figure S1). Student's $t$-test was used to detect the differences between patients with bronchial asthma and healthy subjects, and Kruskal-Wallis test was used to detect the differences among participants with well- or not poor-controlled asthma and healthy subjects in each circadian clock gene expression. Post-hoc analysis was used for comparing the difference among subgroups. Mann-Whitney test was used to detect the differences between asthma patients with and without night symptoms. Receiver operating characteristic (ROC) curve was plotted and ROC area under the curve (AUC) was calculated to compare the discriminating ability of each circadian clock genes. Binary Logistic regression with multivariable analysis modelled the effects of selected independent variables on whether the expression of a specific circadian clock gene could be a predictive marker for asthma or not. The values of $\Delta \mathrm{C} t$ or $-\Delta \mathrm{C} t$ were used for all statistical analysis. All tests were two-sided with statistical significance set at 0.05 .

\section{Results}

\section{Characteristics of Study Participants}

The characteristics, including gender, age, smoking habits, body weight, BMI, body fat, body height, and waist of the 120 patients with bronchial asthma and 60 healthy individuals are listed in Table 1. All the characteristics are not different between patients and healthy individuals. The values for IgE, FEV1, PEF, and ACT score are also listed for the patients with bronchial asthma. The characteristics of well- and not well-controlled asthmatics and asthmatics with and without night symptoms are listed in Table 2.

\section{Expression of Circadian Clock Genes in Patients with Bronchial Asthma}

In order to explore whether expression of circadian clock genes altered in patients with bronchial asthma, we performed qRTPCR to examine the expression of a panel of nine human circadian clock genes in the PBMCs. We found significant difference of expression of all the nine circadian clocks genes 
Table I Characteristics of Study Participants

\begin{tabular}{|c|c|c|c|}
\hline Characteristics & $\begin{array}{l}\text { Healthy Individuals } \\
\qquad(n=60)\end{array}$ & $\begin{array}{c}\text { Bronchial Asthma } \\
\qquad(n=\mid 20)\end{array}$ & $P$-value \\
\hline Gender (Male/Female) & $30 / 30$ & $60 / 60$ & 1.0000 \\
\hline Age, years & $50.98 \pm 13.07$ & $50.63 \pm 12.07$ & 0.8586 \\
\hline Smoking (None/Ever-smoking) & $47 / 13$ & $75 / 45$ & 0.4128 \\
\hline Body Weight, Kg & $65.49 \pm 14.06$ & $68.24 \pm 13.75$ & 0.2108 \\
\hline BMI & $24.34 \pm 3.00$ & $25.74 \pm 4.53$ & 0.1100 \\
\hline Body Fat, \% & $27.76 \pm 8.51$ & $29.32 \pm 12.00$ & 0.3696 \\
\hline Body Height, cm & $164.30 \pm 9.76$ & $162.61 \pm 7.17$ & 0.1918 \\
\hline Waist, cm & $82.05 \pm 10.48$ & $86.25 \pm 16.79$ & 0.0780 \\
\hline $\operatorname{lgE}, \mathrm{IU} / \mathrm{mL}$ & & $330.07 \pm 543.39$ & \\
\hline FEVI, L & & $2.34 \pm 0.72$ & \\
\hline $\mathrm{PEF}, \mathrm{mL} / \mathrm{min}$ & $327.5| \pm| 28.21$ & $369.78 \pm 97.63$ & 0.0658 \\
\hline ACT score & & $20.05 \pm 3.67$ & \\
\hline
\end{tabular}

Notes: Age, BMI, body weight, body height, body fat, waist, IgE, FEVI, PEF, ACT score are expressed as mean standard deviation. The $P$-values indicated were evaluated with Student's $t$-test, except for sex and smoking (Chi-square).

Abbreviations: BMI, body mass index; FEVI, forced expiratory volume in I second; PEF, peak expiratory flow rate; ACT, asthma control test.

between asthmatic patients and healthy individuals (Figure 1A). Except for $B M A L 1$, the expression of the other eight genes was down-regulated in patients with bronchial asthma.
We divided the patients into with and without controlled status, but the expression of all the nine circadian clock genes was not different between these two groups of patients (Figure 1B).

Table 2 Characteristics of Well- and Not Well-Controlled Asthmatics and Asthmatics with and without Night Symptoms

\begin{tabular}{|c|c|c|c|c|c|c|}
\hline Characteristics & $\begin{array}{c}\text { Well- } \\
\text { Controlled } \\
(n=60)\end{array}$ & $\begin{array}{c}\text { Not Well- } \\
\text { Controlled } \\
(n=60)\end{array}$ & $P$-value & $\begin{array}{l}\text { With Night } \\
\text { Symptoms } \\
\quad(n=53)\end{array}$ & $\begin{array}{l}\text { Without Night } \\
\text { Symptoms } \\
(n=67)\end{array}$ & $P$ - value \\
\hline Gender (Male/Female) & $28 / 32$ & $32 / 28$ & 1.000 & $27 / 26$ & $34 / 33$ & 0.996 \\
\hline Age, years & $51.02 \pm 11.67$ & $50.25 \pm 12.54$ & 0.575 & $49.55 \pm|2.5|$ & $51.49 \pm 11.73$ & 0.667 \\
\hline Smoking (None/Ever-smoking) & $43 / 17$ & $33 / 27$ & $0.010 *$ & $30 / 23$ & $45 / 22$ & 0.056 \\
\hline BMI & $25.28 \pm 3.94$ & $26.20 \pm 5.03$ & 0.345 & $25.99 \pm 5.20$ & $25.55 \pm 3.94$ & 0.454 \\
\hline Body Fat, \% & $31.56 \pm 10.99$ & $30.7 I \pm 8.55$ & 0.296 & $25.6 I \pm 4.70$ & $25.49 \pm 3.82$ & 0.225 \\
\hline Waist, cm & $86.00 \pm 10.94$ & $89.48 \pm 13.89$ & 0.157 & $87.28 \pm 12.52$ & $87.10 \pm 11.13$ & 0.312 \\
\hline IgE, IU/mL & $279.59 \pm 482.72$ & $380.56 \pm 602.33$ & 0.307 & $398.12 \pm 606.30$ & $266.56 \pm 479.04$ & 0.282 \\
\hline FEVI, L & $2.37 \pm 0.72$ & $2.30 \pm 0.74$ & 0.610 & $2.29 \pm 0.75$ & $2.39 \pm 0.70$ & 0.818 \\
\hline $\mathrm{PEF}, \mathrm{mL} / \mathrm{min}$ & $369 .|3 \pm 93.7|$ & $370.43 \pm 103.51$ & 0.400 & $366.08 \pm 102.90$ & $373.49 \pm 94.22$ & 0.545 \\
\hline ACT score & $23.10 \pm 1.41$ & $17.00 \pm 0.50$ & $0.020 *$ & $18.69 \pm 3.35$ & $21.12 \pm 3.57$ & 0.222 \\
\hline Night Symptoms, n (\%) & 44 (73.3\%) & $23(38.3 \%)$ & $0.009 * *$ & $53(100 \%)$ & $0(0 \%)$ & - \\
\hline
\end{tabular}

Note: Age, BMI, body fat, waist, IgE, FEVI, PEF, ACT score are expressed as mean standard deviation. The $P$-values indicated were evaluated with Student's $t$-test, except for sex, smoking and night symptoms (Chi-square).

Abbreviations: BMI, body mass index; FEVI, forced expiratory volume in I second; PEF, peak expiratory flow rate; ACT, asthma control test. 


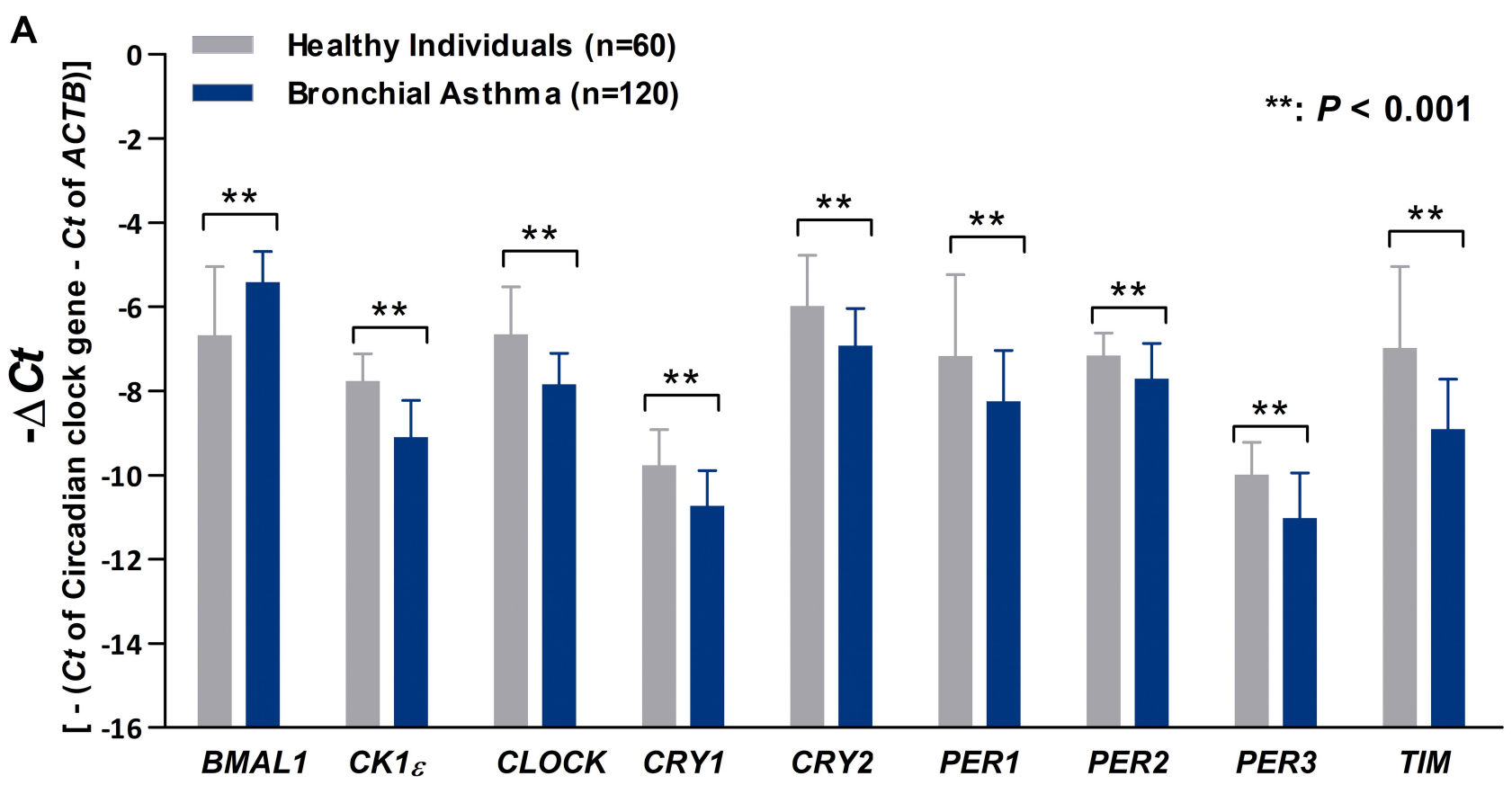

B

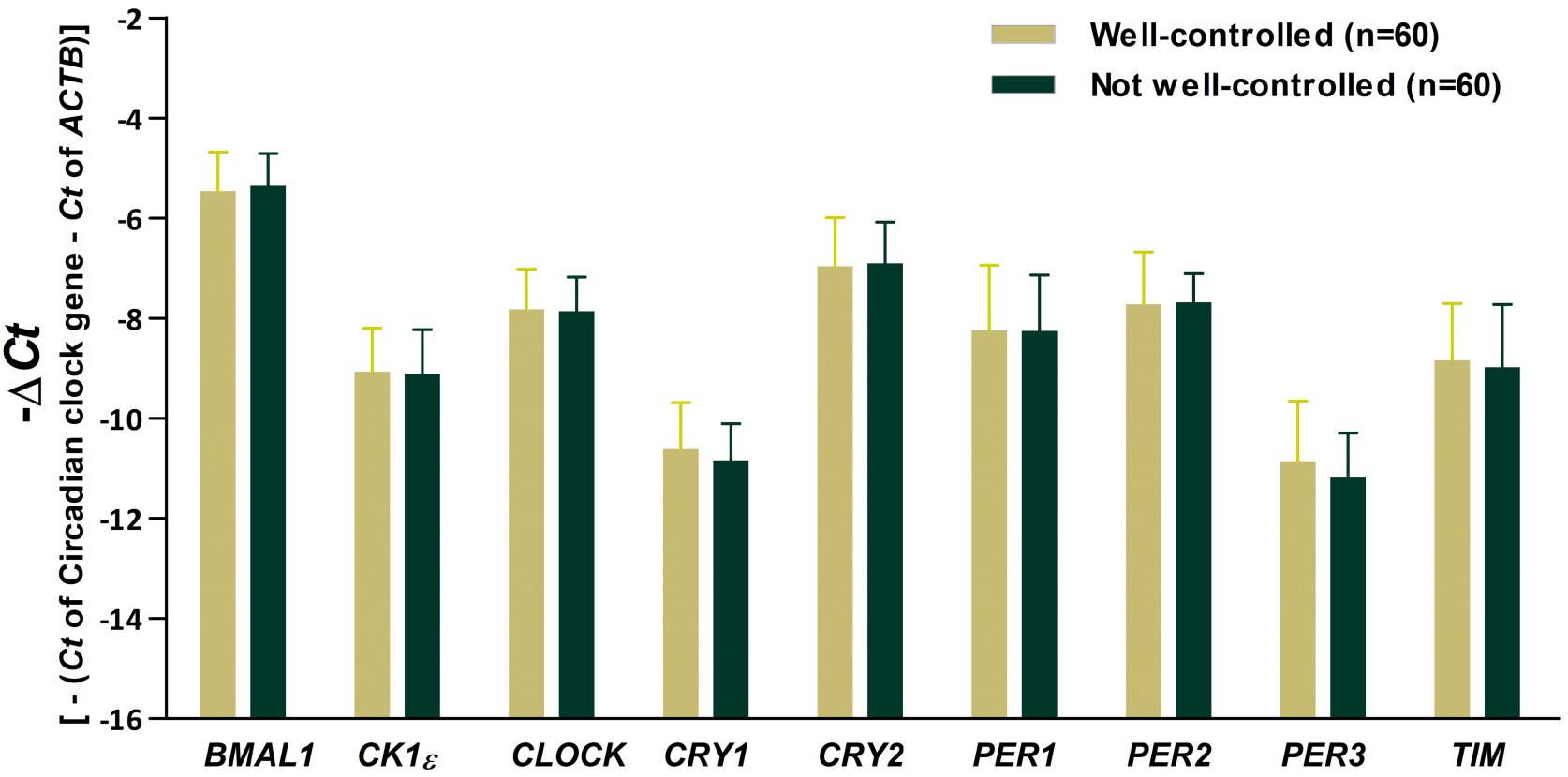

Figure I The expression of nine circadian clock genes in 120 patients with bronchial asthma and 60 healthy individuals as determined by real-time quantitative reverse transcriptase polymerase chain reaction. (A) All nine genes were significantly deregulated in patients with bronchial asthma $(P<0.00 \mathrm{I})$. (B) The expression of all nine circadian clock genes was not different between patients with and without controlled of bronchial asthma. Data presented are mean \pm standard deviation of $-\Delta \mathrm{C} t[-(\mathrm{C} t$ of Circadian clock gene $-\mathrm{C} t$ of $A C T B$ gene)]. Higher $-\Delta C t$ represents higher expression level, and vice versa.

\section{Expression of Circadian Clock Genes in} Asthmatic Patients with and without

\section{Night Symptoms}

To investigate whether the expression of circadian clock genes correlated with night symptoms, we further divided the asthmatic patients into with and without night symptoms groups and performed a Mann-Whitney test to investigate the differential expression of circadian clock genes between these two groups. Among the nine genes, PER3 was the only gene with lower expression in the group with night symptoms $(P=0.0408)$ (Figure 2 and Supplementary Table S1). 


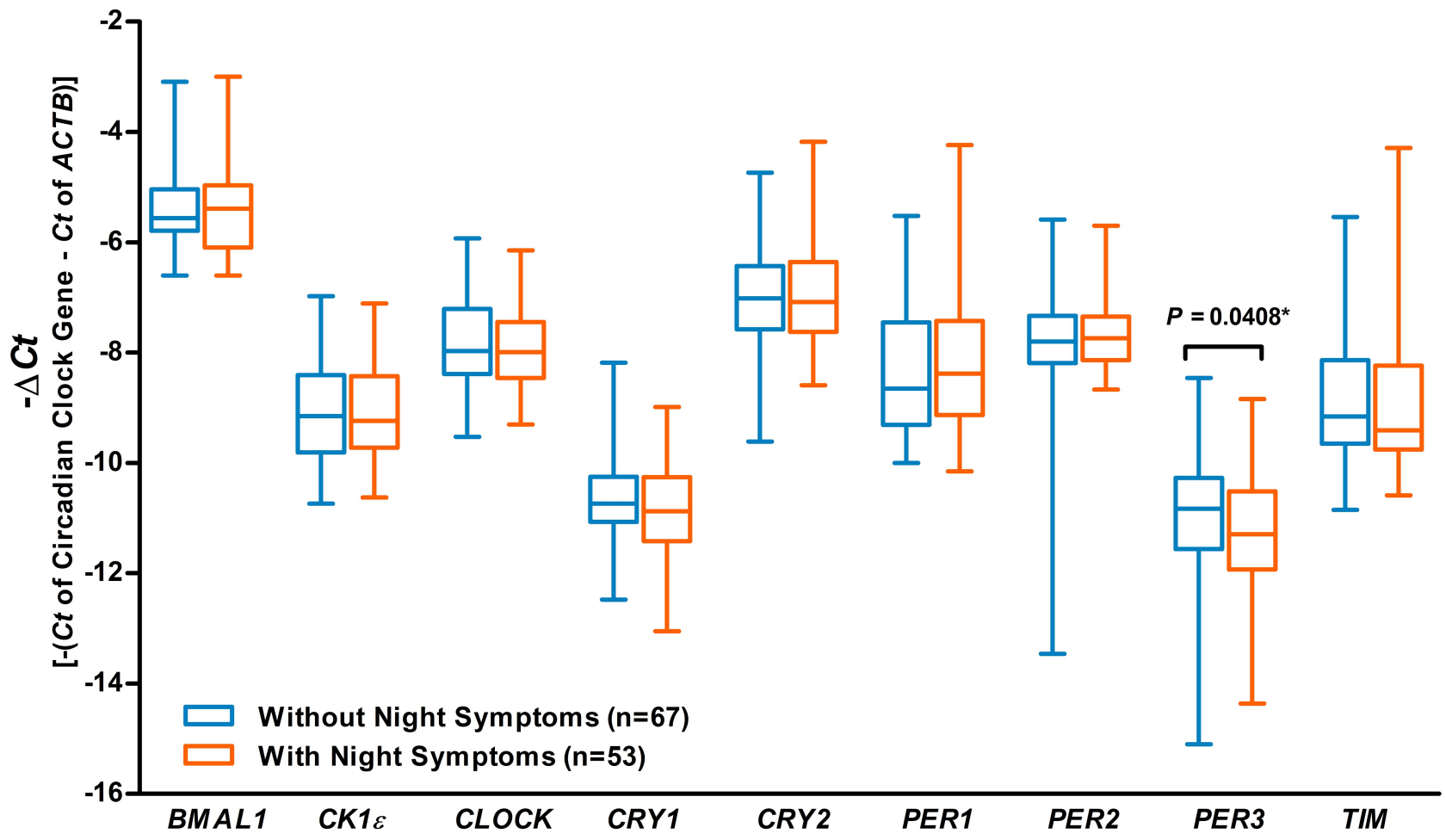

Figure 2 The expression of the nine circadian clock genes in asthmatic patients with and without night symptoms. Expression of PER3 was significantly down-regulated in asthmatic patients with night symptoms as evaluated by Mann-Whitney test using values of $-\Delta C t[-(C t$ of circadian clock gene $-\mathrm{C} t$ of $A C T B$ gene $)$. Data presented are median and range of $-\Delta \mathrm{Ct}$. Higher $-\Delta \mathrm{C}$ t represents higher expression level, and vice versa. $* P<0.05$.

\section{Expression of Circadian Clock Genes in Well- or Not Well-Controlled Asthmatics with and without Night Symptoms}

We further divided the well-controlled asthmatics into groups of with and without night symptoms and found significant down-regulation of BMAL1, CK1E, CLOCK, $C R Y 1, C R Y 2$, and PER1 in the group with night symptoms (Figure 3A and Supplementary Table S2). We also divided the not well-controlled asthmatics into with and without night symptoms groups, and altered expression of BMAL1, CK1E, PER1, and PER2 was observed between these two groups (Figure 3B and Supplementary Table S2).

\section{Predictive Ability for Bronchial Asthma Based on the Expression of Circadian Clock Genes}

To investigate the discriminative performance of expression of circadian clock genes between the groups of asthma and healthy individuals, we constructed ROC curves and calculated the AUC for each gene. Among the nine circadian clock genes, binary logistic regression analysis selected BMAL1 (Odds ratio $=0.23 ; 95 \%$ confidence interval $(\mathrm{CI})$
$=0.12-0.43 ; P<0.0001), C K I \varepsilon \quad($ Odds ratio $=12.34 ; 95 \%$ $\mathrm{CI}=3.92-38.77 ; P<0.0001$ ), $P E R 3$ (Odds ratio=2.15; $95 \%$ $\mathrm{CI}=1.08-4.26 ; P=0.0293$ ), and $T I M$ (Odds ratio=0.45; $95 \% \mathrm{CI}=0.45-0.82 ; P=0.0097)$ as independent factors for bronchial asthma (Table 3). Combined expression of BMAL1, CKIE, PER3, and TIM enhanced the prediction of bronchial asthma (Odds ratio $=624.52 ; 95 \% \mathrm{CI}=115.38-$ 3,380.37; $P<0.0001$ ) (Table 3 ). We also performed ROC analysis and evaluated the AUC of ROC curves to investigate whether patients with bronchial asthma could be distinguished from healthy subjects based on their expression of circadian clock genes (Figure 4). The discriminating predictive abilities of CKIE and four combinations were found to be excellent ( $\mathrm{AUC}=0.879$ and 0.924 , respectively), and predictive abilities of $B M A L 1, P E R 3$, and TIM were acceptable (AUC range=0.683-0.798). Our results demonstrated the combined expression of BMAL1, CKIE, PER3, and TIM might have the potential to be a predictor for bronchial asthma.

\section{Discussion}

In this case-controlled study, we found the expression of all the nine circadian clock genes was altered when 
A

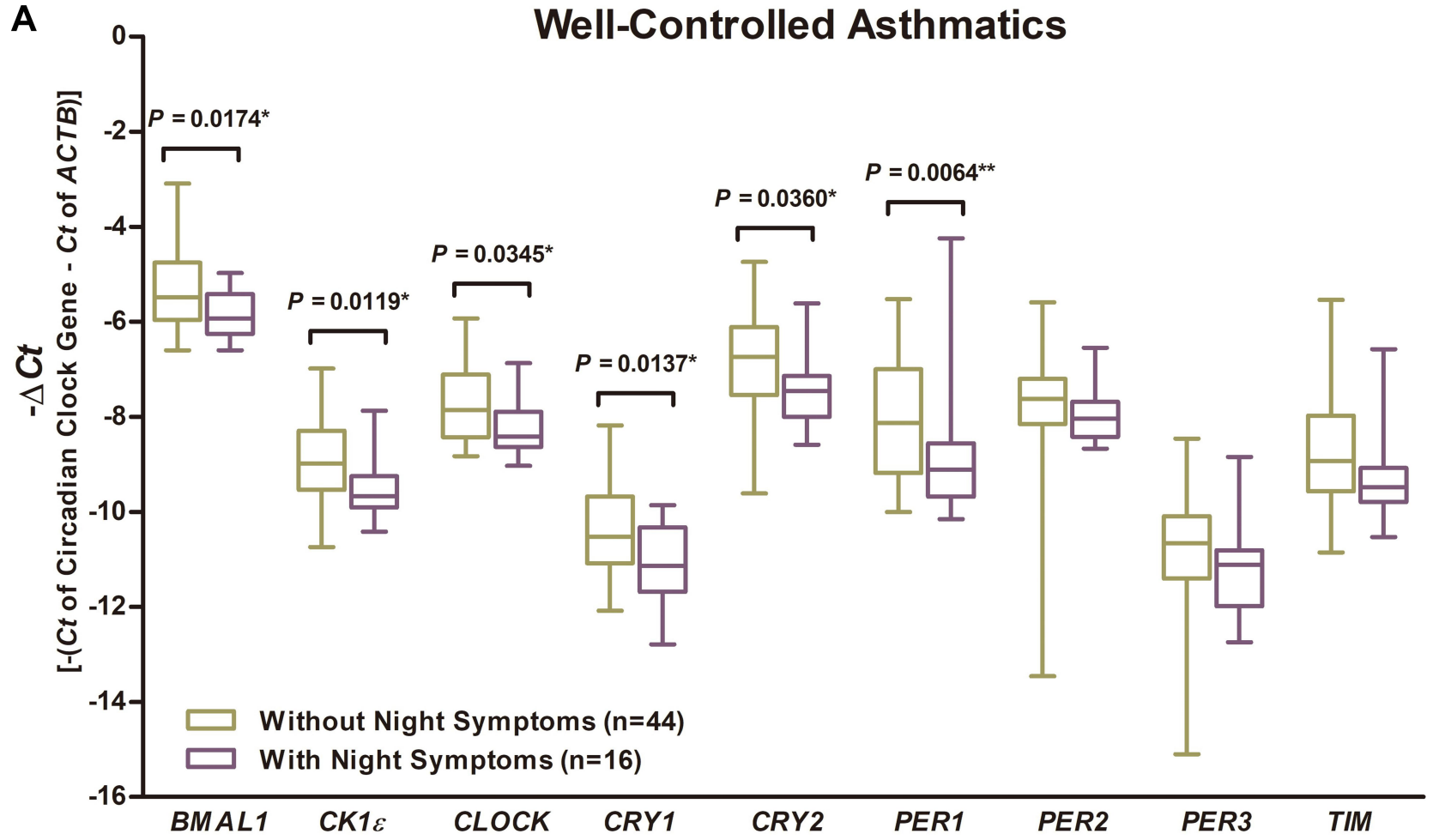

B

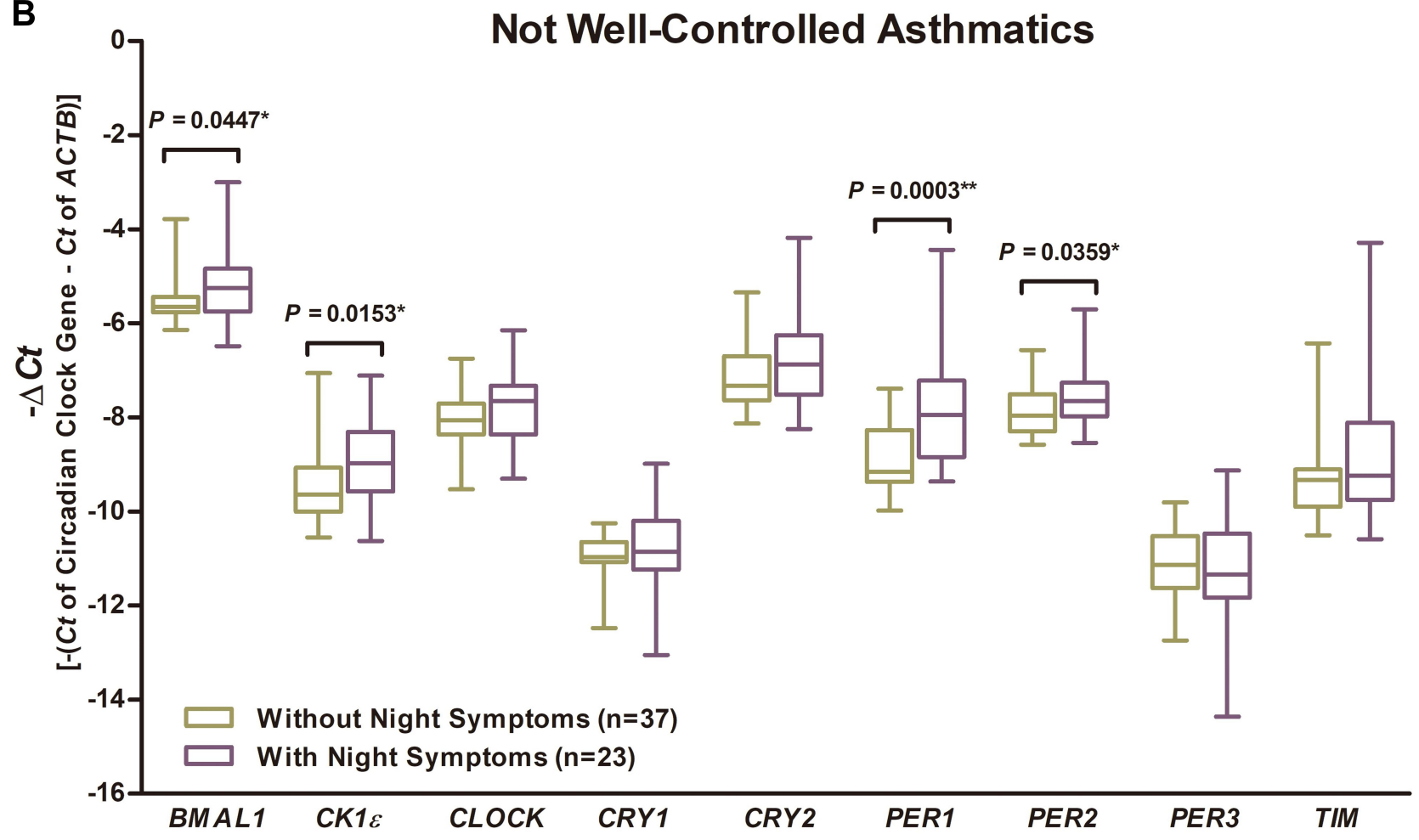

Figure 3 The expression of nine circadian clock genes in patients with and without control of bronchial asthma and with or without night symptoms. (A) In patients with well-controlled bronchial asthma, the expression of BMALI, CKIE, CLOCK, CRYI, CRY2, and PERI was down-regulated in those with night symptoms compared with those without night symptoms. (B) In patients with not well-controlled bronchial asthma, the expression of BMALI, CKIE, PERI, and PER2 was significantly different in those with night symptoms compared with those without night symptoms. Data presented are median and range of $-\Delta \mathrm{Ct}$ [-(Ct of Circadian clock gene $-\mathrm{Ct}$ of $A C T B$ gene)]. Higher $\Delta C t$ represents a higher expression level, and vice versa. $* P<0.05$ and $* * P<0.01$ were evaluated by Mann-Whitney test using values of $-\Delta C t$. 
Table 3 Binary Logistic-Regression Analysis of Predictors of Bronchial Asthma by Expression of BMALI, CKIE, PER3, and TIM.

\begin{tabular}{|l|c|c|c|c|}
\hline Independent Factor & Coefficient & SE & Odds Ratio (95\% Cl) & $P$-value \\
\hline BMALI & -1.48 & 0.32 & $0.23(0.12-0.43)$ & $<0.000 I$ \\
\hline CKIE & $2.5 I$ & 0.58 & $12.34(3.92-38.77)$ & $<0.000 I$ \\
\hline PER3 & 0.76 & 0.35 & $2.15(1.08-4.26)$ & 0.0293 \\
\hline TIM & -0.79 & 0.31 & $0.45(0.25-0.82)$ & 0.0097 \\
\hline Constant & -13.55 & 3.18 & $624.52(115.38-3,380.37)$ & $<0.000 I$ \\
\hline Four combination & & & & \\
\hline
\end{tabular}

Note: The reference for the odds ratio is the absence of the corresponding risk factor.

Abbreviations: $\mathrm{SE}$, standard error; $\mathrm{Cl}$, confidence interval.

compared with that in health individuals. In consistent with our finding, a recent study demonstrated significantly decreased expression of TIM in the blood of children with asthma as compared to the healthy children. ${ }^{28}$ Other than this report on the TIM gene, there have so far been no

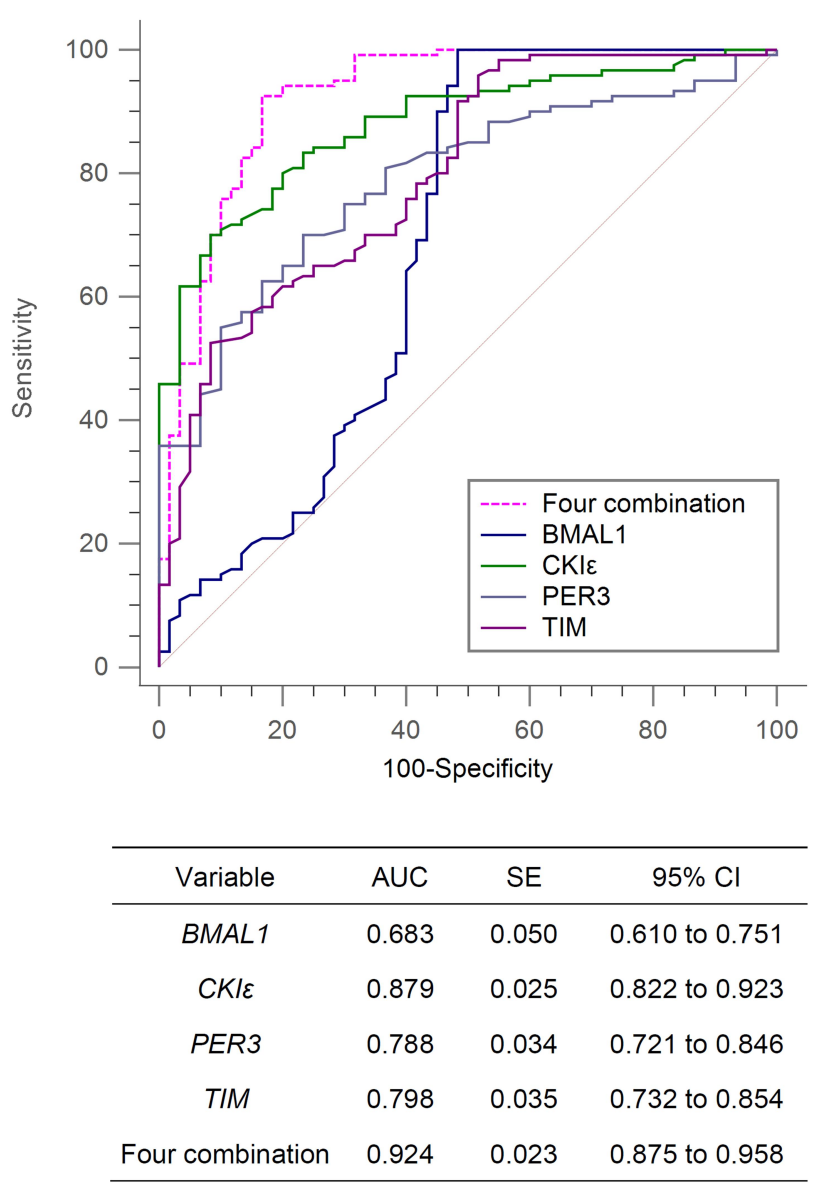

Figure 4 Receiver operator curves demonstrate the predictive ability for bronchial asthma based on the expression of BMALI, CKIE, PER3, TIM, and four combination. Abbreviations: AUC, area under curve; SE, standard error; $\mathrm{Cl}$, confidence interval. reports to directly link the deregulated circadian clock genes and bronchial asthma in humans. Obstructive sleep apnea (OSA) has been reported to be prevalent in nocturnal asthma. ${ }^{29}$ Our previous study indeed observed expressions of eight circadian clock genes (except PER1) at midnight were significantly downregulated in patients with severe OSA. ${ }^{30}$ This may imply the disruption of circadian rhythm caused altered lung function in both asthma and OSA. Deregulated Per2 and Bmall have been associated with inflammation or exacerbated immune function and lung functions in animal models. Recently, a study had disclosed that aberrant light/dark situation aggravated virus-induced, asthma-like inflammation. ${ }^{31}$ Bmall-deleted mice were more susceptible to bacterial infections and inflammation, ${ }^{12,31,32}$ and developed more extensive airway inflammation than wild-type mice. ${ }^{33}$ Inflammatory factors could affect peripheral clock activity and the phase, period, and amplitude of expression of circadian clock genes in normal lung tissue were changed by exposure to cigarette smoke, viral or bacterial infection. ${ }^{34,35}$ In addition, hormonal or autonomic nerve activity synchronized peripheral clocks to the suprachiasmatic nucleus rhythm and affect the asthma activity. ${ }^{36}$ The aberrant hormone secretion or autonomic nerve activity might desynchronize peripheral clock rhythm and might affect the airway activity and allergic reaction. For example, chronic stress would be relating to arrhythmicity in Per2 expression in mice. ${ }^{36}$ Per2 gene regulates daily IL-1 $\beta$ production in macrophages and IFN- $\gamma$ production in NK cells, altered Per 2 gene thus leads to the impairment of immune function. ${ }^{35}$ The majority of asthmatic patients have an allergy reaction and several studies showed the circadian disruption can promote allergy. ${ }^{24,37-39}$ The mechanism might relate to circadian gating of epithelial 
barrier function and immune responses that evolved to complex environmental physical, chemical, and biological stimulants and enhance host defense after exposure of stimulants. $^{24,40}$

In this study, we noted a lower expression of PER3 in asthmatic patients with night symptoms. PER3 was thought to be less important than the other two family members, PER 1 and PER2, in regulating the circadian clock. However, down-regulation of PER3 has been reported in various types of human cancers, ${ }^{26,27,41,42}$ and has been associated with sleep homeostasis and mental disorders in humans. Interestingly, PER3 was also the most down-regulated gene at midnight in OSA and was selected for predicting OSA. ${ }^{30}$ Hence, our finding may add evidence to strengthen the crucial role of PER3 in fine adjustment of response in the time-keeping system in humans.

Another novel finding of this study was the combined expression of BMAL1, CKIE, PER3, and TIM has the potential to predict bronchial asthma. Although depending solely on the expression of circadian clock genes may not be sufficient to predict asthma, they did provide a new reference for evaluating asthma.

Although we have obtained novel and clinically relevant results that could give an insight into a more profound understanding of asthma, there are limitations of this study. First, all the PB samples were obtained in the daytime and only at one time point and therefore the oscillation of circadian clocks genes may not be reflected fully. Second, we did not collect cases at different treatment times to validate whether the changes of gene expression related to the disease status of patients. In addition, this is a correlation study, although we have linked circadian clock genes to with or without control and night symptoms of patients with asthma, no mechanisms of these gene functions were investigated. Due to this study is a retrospective study, we lack detailed medication, treatment response and some data, such as fractional exhaled nitric oxide (FeNO) and allergic profile. Moreover, for the retrospective study with residual samples, we did not have samples to verify our qPCR data at the protein level using either Western blot or immunohistochemical staining.

\section{Conclusion}

This is the first study that directly links circadian clock genes to human asthma. We demonstrated expression levels of the nine core circadian clock genes were altered in patients with bronchial asthma. We also discovered PER3 could be a new reference for evaluating the asthmatics patients with nocturnal symptoms and the combined expression of BMAL1, $C K I \varepsilon, P E R 3$, and TIM has the potential to be a predictor for asthma. Our results may not be sufficient for clinical diagnosis of asthma presently, but they did provide new directions for future studies. Investigating the mechanisms of downregulated circadian clock genes in asthma and the molecular causal effects of circadian clock genes and asthma will also let us take a step forward to reveal the mysterious roles circadian clock genes play in asthma.

\section{Acknowledgments}

This study was funded by grants from the CGMH (Grant number: CMRPG8J0191). The authors thank the Biostatistics Center, K-CGMH for statistics work.

\section{Disclosure}

The authors report no conflicts of interest in this work.

\section{References}

1. Reddel HK, Bateman ED, Becker A, et al. A summary of the new GINA strategy: a roadmap to asthma control. Eur Respir J. 2015;46 (3):622-639. doi:10.1183/13993003.00853-2015

2. Clark TJ. Diurnal rhythm of asthma. Chest. 1987;91(6 Suppl):137S141S. doi:10.1378/chest.91.6_Supplement.137S

3. Litinski M, Scheer FA, Shea SA. Influence of the circadian system on disease severity. Sleep Med Clin. 2009;4(2):143-163. doi:10.1016/j. jsmc.2009.02.005

4. Martin RJ. Location of airway inflammation in asthma and the relationship to circadian change in lung function. Chronobiol Int. 1999;16(5):623-630. doi:10.3109/07420529908998731

5. Sutherland ER. Nocturnal asthma. J Allergy Clin Immunol. 2005;116 (6):1179-1186; quiz 1187. doi:10.1016/j.jaci.2005.09.028

6. Albrecht U. Timing to perfection: the biology of central and peripheral circadian clocks. Neuron. 2012;74(2):246-260. doi:10.1016/j. neuron.2012.04.006

7. Mohawk JA, Green CB, Takahashi JS. Central and peripheral circadian clocks in mammals. Annu Rev Neurosci. 2012;35:445-462. doi:10.1146/annurev-neuro-060909-153128

8. Greenberg H, Cohen RI. Nocturnal asthma. Curr Opin Pulm Med. 2012;18(1):57-62. doi:10.1097/MCP.0b013e32834d098e

9. Sundar IK, Yao H, Sellix MT, Rahman I. Circadian molecular clock in lung pathophysiology. Am J Physiol Lung Cell Mol Physiol. 2015;309(10):L1056-L1075. doi:10.1152/ajplung.00152.2015

10. Silver AC, Arjona A, Walker WE, Fikrig E. The circadian clock controls toll-like receptor 9-mediated innate and adaptive immunity. Immunity. 2012;36(2):251-261. doi:10.1016/j.immuni.2011.12.017

11. Gibbs JE, Beesley S, Plumb J, et al. Circadian timing in the lung; a specific role for bronchiolar epithelial cells. Endocrinology. 2009;150(1):268-276. doi:10.1210/en.2008-0638

12. Pekovic-Vaughan V, Gibbs J, Yoshitane H, et al. The circadian clock regulates rhythmic activation of the NRF2/glutathione-mediated antioxidant defense pathway to modulate pulmonary fibrosis. Genes Dev. 2014;28(6):548-560. doi:10.1101/gad.237081.113 
13. Keller M, Mazuch J, Abraham U, et al. A circadian clock in macrophages controls inflammatory immune responses. Proc Natl Acad Sci USA. 2009;106(50):21407-21412. doi:10.1073/pnas.0906361106

14. Gibbs JE, Blaikley J, Beesley S, et al. The nuclear receptor REV$\mathrm{ERB} \alpha$ mediates circadian regulation of innate immunity through selective regulation of inflammatory cytokines. Proc Natl Acad Sci USA. 2012;109(2):582-587. doi:10.1073/pnas.1106750109

15. Hayashi M, Shimba S, Tezuka M. Characterization of the molecular clock in mouse peritoneal macrophages. Biol Pharm Bull. 2007;30 (4):621-626. doi:10.1248/bpb.30.621

16. Born J, Lange T, Hansen K, Molle M, Fehm HL. Effects of sleep and circadian rhythm on human circulating immune cells. J Immunol. 1997;158(9):4454-4464.

17. Rahman SA, Castanon-Cervantes O, Scheer FA, et al. Endogenous circadian regulation of pro-inflammatory cytokines and chemokines in the presence of bacterial lipopolysaccharide in humans. Brain Behav Immun. 2015;47:4-13. doi:10.1016/j.bbi.2014.11.003

18. Fernandes G, Halberg F, Yunis EJ, Good RA. Circadian rhythmic plaque-forming cell response of spleens from mice immunized with SRBC. J Immunol. 1976;117(3):962-966.

19. Bollinger T, Leutz A, Leliavski A, et al. Circadian clocks in mouse and human CD4+ T cells. PLoS One. 2011;6(12):e29801. doi:10.1371/journal.pone.0029801

20. Silver AC, Arjona A, Hughes ME, Nitabach MN, Fikrig E. Circadian expression of clock genes in mouse macrophages, dendritic cells, and B cells. Brain Behav Immun. 2012;26(3):407-413. doi:10.1016/j. bbi.2011.10.001

21. Kelly EA, Houtman JJ, Jarjour NN. Inflammatory changes associated with circadian variation in pulmonary function in subjects with mild asthma. Clin Exp Allergy. 2004;34(2):227-233. doi:10.1111/j.13652222.2004.01866.x

22. Christ P, Sowa AS, Froy O, Lorentz A. The circadian clock drives mast cell functions in allergic reactions. Front Immunol. 2018;9:1526. doi:10.3389/fimmu.2018.01526

23. Nakao A. Clockwork allergy: how the circadian clock underpins allergic reactions. J Allergy Clin Immunol. 2018;142(4):1021-1031. doi:10.1016/j.jaci.2018.08.007

24. Nakao A. Circadian regulation of the biology of allergic disease: clock disruption can promote allergy. Front Immunol. 2020;11:1237. doi:10.3389/fimmu.2020.01237

25. Zasłona Z, Case S, Early JO, et al. The circadian protein BMAL1 in myeloid cells is a negative regulator of allergic asthma. Am J Physiol Lung Cell Mol Physiol. 2017;312(6):L855-860. doi:10.1152/ ajplung.00072.2017

26. Yang MY, Chang JG, Lin PM, et al. Downregulation of circadian clock genes in chronic myeloid leukemia: alternative methylation pattern of hPER3. Cancer Sci. 2006;97(12):1298-1307. doi:10.1111/j.1349-7006.2006.00331.x

27. Yang MY, Yang WC, Lin PM, et al. Altered expression of circadian clock genes in human chronic myeloid leukemia. J Biol Rhythms. 2011;26(2):136-148. doi:10.1177/0748730410395527
28. Langwinski W, Sobkowiak P, Narozna B, et al. Association of circadian clock TIMELESS variants and expression with asthma risk in children. Clin Respir J. 2020;14(12):1191-1200. doi:10.1111/crj.13260

29. Shigemitsu H, Afshar K. Nocturnal asthma. Curr Opin Pulm Med. 2007;13(1):49-55. doi:10.1097/MCP.0b013e328010a890

30. Yang MY, Lin PW, Lin HC, et al. Alternations of circadian clock genes expression and oscillation in obstructive sleep apnea. J Clin Med. 2019;8(10):1634. doi:10.3390/jcm8101634

31. Ehlers A, Xie W, Agapov E, et al. BMAL1 links the circadian clock to viral airway pathology and asthma phenotypes. BMAL1 links the circadian clock to viral airway pathology and asthma phenotypes. Mucosal Immunol. 2018;11(1):97-111. doi:10.1038/mi.2017.24

32. Nguyen KD, Fentress SJ, Qiu Y, Yun K, Cox JS, Chawla A. Circadian gene Bmal1 regulates diurnal oscillations of Ly6C(hi) inflammatory monocytes. Science. 2013;341(6153):1483-1488. doi: $10.1126 /$ science. 1240636

33. Gibbs J, Ince L, Matthews L, et al. An epithelial circadian clock controls pulmonary inflammation and glucocorticoid action. Nat Med. 2014;20(8):919-926. doi:10.1038/nm.3599

34. Hwang JW, Sundar IK, Yao H, Sellix MT, Rahman I. Circadian clock function is disrupted by environmental tobacco/cigarette smoke, leading to lung inflammation and injury via a SIRT1-BMAL1 pathway. FASEB J. 2014;28(1):176-194. doi:10.1096/fj.13-232629

35. Annamneedi VP, Park JW, Lee GS, Kang TJ. Cell autonomous circadian systems and their relation to inflammation. Biomol Ther (Seoul). 2021;29(1):31-40. doi:10.4062/biomolther.2020.215

36. Nakamura Y, Ishimaru K, Tahara Y, Shibata S, Nakao A. Disruption of the suprachiasmatic nucleus blunts a time of day-dependent variation in systemic anaphylactic reaction in mice. $J$ Immunol Res. 2014;2014:474217. doi:10.1155/2014/474217

37. Kawauchi T, Ishimaru K, Nakamura Y, et al. Clock-dependent temporal regulation of IL-33/ST2-mediated mast cell response. Allergol Int. 2017;66(3):472-478. doi:10.1016/j.alit.2017.02.004

38. Nakamura Y, Nakano N, Ishimaru K, et al. Circadian regulation of allergic reactions by the mast cell clock in mice. J Allergy Clin Immunol. 2014;133(2):568-575. doi:10.1016/j.jaci.2013.07.040

39. Ihara T, Mitsui $T$, Nakamura $Y$, et al. Clock genes regulate the circadian expression of Piezo1, TRPV4, Connexin26, and VNUT in an ex vivo mouse bladder mucosa. PLoS One. 2017;12(1):e0168234. doi:10.1371/journal.pone.0168234

40. Curtis AM, Bellet MM, Sassone-Corsi P, O’Neill LA. Circadian clock proteins and immunity. Immunity. 2014;40(2):178-186. doi:10.1016/j.immuni.2014.02.002

41. Yang MY, Lin PM, Hsiao HH, et al. Up-regulation of PER3 expression is correlated with better clinical outcome in acute leukemia. Anticancer Res. 2015;35(12):6615-6622.

42. Hsu CM, Lin SF, Lu CT, Lin PM, Yang MY. Altered expression of circadian clock genes in head and neck squamous cell carcinoma. Tumour Biol. 2012;33(1):149-155.

\section{Publish your work in this journal}

The Journal of Asthma and Allergy is an international, peer-reviewed open-access journal publishing original research, reports, editorials and commentaries on the following topics: Asthma; Pulmonary physiology; Asthma related clinical health; Clinical immunology and the immunological basis of disease; Pharmacological interventions and new therapies. The manuscript management system is completely online and includes a very quick and fair peer-review system, which is all easy to use. Visit http://www.dovepress.com/testimonials.php to read real quotes from published authors. 